\title{
AVALIAÇÃO DO CRESCIMENTO INICIAL DE FRUTÍFERAS EM SISTEMA AGROFLORESTAIS NO P. A. BELO HORIZONTE I, SÃO DOMINGOS DO ARAGUAIA, PA
}

\author{
Tatiane Pereira Guimarães ${ }^{1}$; Rosana Quaresma Maneschy² ${ }^{2}$ Ilmaione Keiza de Souza \\ Oliveira $^{3}$; Karolinny Carneiro Guerra Costa ${ }^{4}$; Albinei Araujo de Castro ${ }^{5}$ \\ ${ }^{1}$ Discente de Agronomia, Faculdade de Ciências Agrárias de Marabá (FCAM), Universidade Federal do Pará (UFPA), \\ Bolsista FAPESPA, tatianepg.ufpa@gmail.com \\ ${ }^{2}$ Prof. a , DSc., FCAM, UFPA, romaneschy@ufpa.br \\ ${ }^{3}$ Discente de Agronomia, FCAM, UFPA, Bolsista PIBIC/PARD, ilmaionekeiza@ hotmail.com \\ ${ }^{4}$ Discente de Agronomia, FCAM, UFPA, Bolsista PIBIC/UFPA, karolinnycg.guerra@gmail.com \\ ${ }^{5}$ Discente de Agronomia, FCAM, UFPA, Bolsista FAPESPA, albinei_araujo@ hotmail.com
}

RESUMO: Tradicionalmente os agroecossistemas de pastagem são monoculturas sem a utilização do componente arbóreo associado. A utilização de sistemas agroflorestais (SAF) pecuários com ênfase nas espécies frutíferas podem ser alternativas viáveis na recuperação de áreas degradadas aliadas a diversificação produtiva e garantia de segurança alimentar para a agricultura familiar. Nesse contexto esse estudo avaliou o crescimento inicial das espécies frutíferas abacate (Persea americana L.), açaí (Euterpe oleracea Mart.), bacuri (Platonia insignis Mart.) e graviola (Annona muricata L.) em SAF, implantados em áreas de pastagens degradadas, de unidades de produção familiares no assentamento rural Belo Horizonte I, localizado no município de São Domingos do Araguaia-PA Os SAF foram implantados em dezembro de 2009 e fevereiro de 2010. O local de implantação da ação-teste e as espécies utilizadas foram escolhidas de forma participativa. As espécies frutíferas açaí e bacuri tiveram a sobrevivência e crescimento comprometidos pelo estresse hídrico durante o período seco do ano. As espécies abacate e graviola se mostraram mais resistentes e, portanto mais indicadas a compor SAF implantados em áreas de pastagens degradadas, nas condições avaliadas nas unidades de produção familiares no PA Belo Horizonte I.

PALAVRAS-CHAVE: Agroecossitemas, frutíferas, crescimento.

\section{EVALUACIÓN DEL CRECIMIENTO INICIAL DE FRUTALES EN SISTEMAS AGROFORESTALES EN EL P. A. BELO HORIZONTE I, SÃO DOMINGOS DO ARAGUAIA, PA}

RESUMEN: Tradicionalmente los agroecosistemas de pasturas son monocultivos, sin la utilización del componente arbóreo asociados. El uso de agroforestales (SAF) pecuario, con énfasis en especies frutales pueden ser una alternativa viable en la recuperación de áreas degradadas, junto con la diversificación de la producción y garantizar la seguridad alimentaria de la granja familiar. En este contexto, este estudio examinó el crecimiento inicial de las especies frutales de aguacate (Persea americana L.), açaí (Euterpe oleracea Mart.), bacuri (Platonia insignis Mart.) y guanábana (Annona muricata L.) en los SAF, implantado en pasturas degradadas de las unidades de producción familiar en los asentamientos rurales que Belo Horizonte, ubicado en São Domingos do 
Araguaia-PA. El SAF fue hecho en diciembre de 2009 y febrero de 2010. El sitio de implantación de las especies de acción y de prueba utilizados fueron elegidos de manera participativa. Las especies frutales açaí y bacuri tuvieran la supervivencia y el crecimiento afectados negativamente por el estrés de agua durante la estación seca. Guanábana y aguacate son especies más resistentes y por lo tanto más apropiado para los SAF establecidos en pasturas degradadas en las condiciones evaluadas en las unidades de producción familiares en el P. A. Belo Horizonte I.

PALABRAS CLAVES: Agroecosistemas, frutales, crecimiento.

\section{INTRODUÇÃO}

Segundo Carvalho (2008), vivemos atualmente um período de crise ecológica, no qual os bens naturais estão cada vez mais escassos, degradados e poluídos. Nos anos recentes, têm sido dadas demonstrações irrefutáveis da insustentabilidade do modelo de exploração da natureza praticado pela nossa civilização.

$\mathrm{Na}$ Amazônia, atualmente, além da agricultura convencional, a expansão da pecuária extensiva vem causando modificações em grandes extensões de florestas nativas. Em razão do manejo inadequado, muitos solos da região tornam-se improdutivos. Os resultados refletem na degradação dos solos, assoreamento de rios, igarapés e destruição de áreas de floresta nativa com uma riqueza de recursos potencialmente úteis para o homem, que podem não ter sido sequer conhecidos $\mathrm{e}$ identificados (FERNANDES; SERRÃO, 1992).
Para tentar mitigar os impactos da degradação das pastagens tem sido considerada pela comunidade científica e pelos produtores a utilização de sistemas agroflorestais (SAF). Os SAF integram cultivos perenes, às essências florestais e/ou fruteiras, também podendo estar associado as pastagens e animais (DUBOIS et al., 1996).

Nesse contexto os sistemas agroflorestais pecuários (SAFPs) são considerados como uma alternativa promissora, para integração de espécies lenhosas a atividades agropastoris, como estratégia de recuperação de áreas de pastagem degradadas (VEIGA et al., 2000). Então para que a região alcance níveis mais sustentáveis de desenvolvimento, é necessário aliar a preservação do ambiente amazônico à garantia de segurança alimentar e geração de emprego e renda para a agricultura familiar. Além disso, a nova política de acesso ao crédito exige a regularização das propriedades em termos da reconstituição das áreas de reserva legal (ARL) e áreas de preservação 
permanente (APP's) para aqueles que estiverem irregulares com a lei ambiental.

A adoção de espécies frutíferas em SAFP contribui para viabilizar a permanência do agricultor em uma mesma área, colaborar com a segurança alimentar, gerar ingressos adicionais de renda, maximizar a produtividade por unidade, propiciar o aumento da biodiversidade, otimizar a absorção de nutrientes e água, recuperar áreas degradadas e/ou abandonadas para que seja possível alcançar sustentabilidade nessas unidades de produção familiar, mediante inovações nesses sistemas de produção e comercialização (OLIVEIRA, 1997)

O trabalho teve como objetivo avaliar o crescimento inicial das espécies frutíferas abacate (Persea americana L.), açaí (Euterpe oleracea Mart.), bacuri (Platonia insignis Mart.) e graviola (Annona muricata L.) em sistemas agroflorestais (SAFs), implantados em áreas de pastagens degradadas, de unidades de produção familiares no Sudeste do Pará.

\section{MATERIAL E MÉTODOS}

A pesquisa foi realizada no âmbito do projeto "Sistemas silvipastoris e agrossilvipastoris como alternativa para a sustentabilidade da pecuária na agricultura familiar da região de Marabá - PA" desenvolvido pela Universidade Federal do
Pará - Campus de Marabá com apoio financeiro da Fundação de Amparo à Pesquisa do Estado do Pará (FAPESPA).

O local da pesquisa foi no P. A. Belo Horizonte I, situado à altura do km 30 da BR153, no Município de São Domingos do Araguaia, na Microrregião de Marabá.

O clima no local segundo a classificação de Köppen é Am no limite de transição para Aw com temperatura média anual de $26,3^{\circ} \mathrm{C}$, caracterizado por um período menos chuvoso entre os meses de maio e outubro e um período mais chuvoso entre os meses de novembro a abril. Solo podzólico vermelho amarelo com textura argilo-arenosa (NAVEGANTES-ALVES, 2009).

A abordagem do projeto se enquadra nas metodologias de pesquisa-ação e de pesquisa participativa em meio real e visam à construção de inovações que serão desenvolvidos em parcerias com os agricultores (CHIA et al., 2006). Considera-se como ação-teste quando guiado por um facilitador os agricultores realizam um pequeno ensaio teste em parcelas pequenas, as quais podem ter diferentes tratamentos (CIAT, 1993).

Nesse contexto foram implantadas ações-testes agroflorestais em três lotes de agricultores familiares (Quadro 1). O local e as espécies componentes dos SAF foram 
escolhidos pelas famílias. As áreas escolhidas tinham como vegetação dominante anteriormente floresta primária, que foram suprimidas há aproximadamente uns 15 anos para formação de pastagem com braquiarão (Brachiaria brizantha cv. marandu Hochst. ex A. Rich). As pastagens foram utilizadas por 10 anos e nos últimos cinco anos estão em pousio.
Para a implantação das ações-teste foram realizadas análises de solo das áreas (Tabela 1). Em todas as ações-teste a implantação das mudas se deu de forma aleatória, não configurando linhas de plantio específicas por espécie. O único trato cultural realizado foi a capina com a finalidade de diminuir a competição entre as espécies introduzidas e a gramínea.

Quadro 1. Ações-testes agroflorestais em três lotes de agricultores familiares no P. A. Belo Horizonte I, São Domingos do Araguaia - PA.

\begin{tabular}{|c|c|c|c|c|c|}
\hline Ação-teste & Implantação & Espécies & $\begin{array}{l}\text { Área } \\
\text { (ha) }\end{array}$ & $\begin{array}{c}\text { Espaçamento } \\
\text { (m) }\end{array}$ & Preparo de área \\
\hline SAF 1 & Dez/2009 & $\begin{array}{l}\text { Bacuri, feijão guandu*, feijão trepa } \\
\text { pau* e milho* e sapucaia }\end{array}$ & 0,65 & $10 \times 5$ & $\begin{array}{l}\text { Aração e } \\
\text { gradagem }\end{array}$ \\
\hline SAF 2 & Fev/2010 & $\begin{array}{l}\text { Abacate, açaí, ipê amarelo, ipê branco } \\
\text { e neem }\end{array}$ & 0,25 & $3 \times 2$ & Roçagem manual \\
\hline SAF 3 & Fev/2010 & $\begin{array}{l}\text { Aroeira, arroz*, favinha, goiaba, } \\
\text { graviola, ipê amarelo, ipê branco, ipê } \\
\text { rosa, ipê roxo, jatobá mandioca*, } \\
\text { neem }\end{array}$ & 0,37 & $10 \times 4$ & $\begin{array}{l}\text { Aração e } \\
\text { gradagem }\end{array}$ \\
\hline
\end{tabular}

* Espécies plantadas nas entrelinhas.

Tabela 1. Características físicas e químicas dos solos nas ações-teste agroflorestais, na camada de 0-20 cm, no P. A. Belo Horizonte I, São Domingos do Araguaia, Pará.

\begin{tabular}{|c|c|c|c|c|c|c|c|c|c|c|c|}
\hline SAF & $\begin{array}{l}\text { Areia } \\
\text { grossa }\end{array}$ & $\begin{array}{l}\text { Areia } \\
\text { fina }\end{array}$ & Silte & $\begin{array}{l}\text { Argila } \\
\text { total }\end{array}$ & $\begin{array}{c}\mathrm{pH} \\
\text { (água) }\end{array}$ & --- & $\mathrm{g} \mathrm{dm}$ & $\mathrm{Na}$ & $\mathrm{Ca}$ & $\begin{array}{c}\mathrm{Ca}+\mathrm{Mg} \\
\mathrm{mol}_{\mathrm{c}} \mathrm{dm}^{-3}\end{array}$ & $\mathrm{Al}$ \\
\hline 1 & 190 & 453 & 194 & 165 & 5,5 & 3 & 47 & 19 & 1,9 & 2,7 & 0,6 \\
\hline 2 & 174 & 529 & 197 & 100 & 5,6 & 5 & 77 & 27 & 2,8 & 3,6 & 0,1 \\
\hline 3 & 191 & 434 & 206 & 170 & 5,8 & 6 & 163 & 62 & 2,8 & 3,8 & 0,1 \\
\hline
\end{tabular}


Para a avaliação das espécies frutíferas foram desconsideradas as fileiras externas de plantas dos três SAFs. As avaliações foram realizadas em dez indivíduos por espécie, sendo elas: taxa de sobrevivência, altura total da planta (m); e diâmetro do coleto (mm) aos 45, 75, 105, 135 e 165 dias após o plantio. Foram testadas equações de regressão para estimar os padrões de crescimento avaliados em relação à idade do plantio. A acurácia dos modelos de regressão foi avaliada por meio do teste $\mathrm{F}$ ao nível de 5\%. Também foram realizadas entrevistas não diretivas com a família para o relato dos tratos culturais efetuados na manutenção das ações-teste agroflorestais.

\section{RESULTADOS E DISCUSSÃO}

Na Tabela 2 estão dispostas os dados de sobrevivência, altura total e diâmetro do coleto das espécies abacate, açaí, bacuri e graviola nos SAF avaliados. No SAF1 a espécie de interesse do agricultor foi o bacuri. As mudas foram plantadas a pleno sol, logo após o preparo do solo, no entanto o agricultor logo em seguida fez uso do plantio das culturas de ciclo curto, que supriram a exigência de sombra da espécie. Mas em meio às condições do agricultor que não possui sistema de irrigação e na maior parte do período menos chuvoso as mudas ficaram em condições de stress hídrico e sem sombra e acabaram morrendo até o fim da avaliação. Segundo Xavier (2001) o bacuri é uma frutífera adaptada a sombra e exigente com água, sendo ainda considera apropriada para a formação de consorcio com outras plantas de porte florestal.

$\mathrm{Na}$ ação-teste agroflorestais do SAF 2 as espécies frutíferas implantadas foram o abacate e açaí. $\mathrm{O}$ crescimento das mudas foi bastante favorável, até que o período seco se intensificou e isso pode ter sido um fator responsável pela morte das mudas de açaí, que é uma espécie exigente em água, refletindo em uma baixa taxa de sobrevivência. Segundo Calzavara (1972) o açaí é uma espécie indicada para as condições tropicais de grande precipitação pluviométrica e elevada temperatura, possibilitando proteção permanente ao solo. $\mathrm{O}$ abacate foi mais resistente a falta de água, e apresentou taxa elevada de sobrevivência (100\%).

No SAF 3 a espécie frutífera avaliada foi à graviola, que apresentou boa taxa de sobrevivência inclusive no período seco, que pode ser explicada por ser uma espécie rústica, resistente ao déficit hídrico e a baixa fertilidade do solo (BARBOSA et al., 2003).

As relações dos parâmetros de crescimento nos períodos avaliados nas três ações-teste agroflorestais foram consideradas significativas $(\mathrm{P} \geq 0,05)$ (Figura 1). 
Tabela 2. Taxa de sobrevivência (S), altura total (AT) e diâmetro do coleto (DCL) do abacate, açaí, bacuri, graviola, nas ações-teste agroflorestais em três lotes de agricultores familiares no P. A. Belo Horizonte I, São Domingos do Araguaia, Pará.

\begin{tabular}{|c|c|c|c|c|c|c|c|c|c|c|c|c|c|c|c|c|}
\hline \multirow{3}{*}{ SAF } & \multirow{3}{*}{ ESP } & \multicolumn{15}{|c|}{ Período de Avaliação (dias) } \\
\hline & & \multicolumn{3}{|c|}{45} & \multicolumn{3}{|c|}{75} & \multicolumn{3}{|c|}{105} & \multicolumn{3}{|c|}{135} & \multicolumn{3}{|c|}{165} \\
\hline & & $\begin{array}{c}\mathrm{S} \\
(\%)\end{array}$ & $\begin{array}{l}\text { AT } \\
(\mathrm{cm})\end{array}$ & $\begin{array}{l}\text { DCL } \\
(\mathrm{mm})\end{array}$ & $\begin{array}{c}\mathrm{S} \\
(\%)\end{array}$ & $\begin{array}{l}\text { AT } \\
(\mathrm{cm})\end{array}$ & $\begin{array}{c}\mathrm{DC} \\
(\mathrm{mm})\end{array}$ & $\begin{array}{c}\mathrm{S} \\
(\%)\end{array}$ & $\begin{array}{l}\text { AT } \\
(\mathrm{cm})\end{array}$ & $\begin{array}{c}\mathrm{DC} \\
(\mathrm{mm})\end{array}$ & $\begin{array}{c}\mathrm{S} \\
(\%)\end{array}$ & $\begin{array}{l}\text { AT } \\
(\mathrm{cm})\end{array}$ & $\begin{array}{c}\mathrm{DC} \\
(\mathrm{mm})\end{array}$ & $\begin{array}{c}\mathrm{S} \\
(\%)\end{array}$ & $\begin{array}{l}\text { AT } \\
(\mathrm{cm})\end{array}$ & $\begin{array}{c}\mathrm{DC} \\
(\mathrm{mm})\end{array}$ \\
\hline 1 & BCR & 70 & $\begin{array}{c}20,71 \\
( \pm 5,68)\end{array}$ & $\begin{array}{c}5,68 \\
( \pm 0,01)\end{array}$ & 70 & $\begin{array}{c}23,57 \\
( \pm 6,45)\end{array}$ & $\begin{array}{c}6,45 \\
( \pm 0,01)\end{array}$ & 50 & $\begin{array}{c}27,00 \\
( \pm 6,52)\end{array}$ & $\begin{array}{c}6,52 \\
( \pm 0,02)\end{array}$ & 50 & $\begin{array}{c}29,00 \\
( \pm 7,35)\end{array}$ & $\begin{array}{c}7,35 \\
( \pm 0,02)\end{array}$ & 0 & - & - \\
\hline 2 & $\mathrm{ABT}$ & 100 & $\begin{array}{c}30,4 \\
( \pm 12,18)\end{array}$ & $\begin{array}{c}0,1 \\
( \pm 0,07)\end{array}$ & 100 & $\begin{array}{c}38 \\
( \pm 11,02)\end{array}$ & $\begin{array}{c}0,14 \\
( \pm 0,11)\end{array}$ & 100 & $\begin{array}{c}45,6 \\
( \pm 10,50)\end{array}$ & $\begin{array}{c}0,2 \\
( \pm 0,08)\end{array}$ & 100 & $\begin{array}{c}45,6 \\
( \pm 10,50)\end{array}$ & $\begin{array}{c}0,27 \\
( \pm 0,37)\end{array}$ & 100 & $\begin{array}{c}48 \\
( \pm 10,11)\end{array}$ & $\begin{array}{c}0,48 \\
( \pm 0,22)\end{array}$ \\
\hline & $\mathrm{ACI}$ & 100 & $\begin{array}{c}20,5 \\
( \pm 0,53)\end{array}$ & $\begin{array}{c}0,04 \\
( \pm 0,00)\end{array}$ & 100 & $\begin{array}{c}25,8 \\
( \pm 3,85)\end{array}$ & $\begin{array}{c}0,06 \\
( \pm 0,01)\end{array}$ & 100 & $\begin{array}{c}30,7 \\
( \pm 2,71)\end{array}$ & $\begin{array}{c}0,09 \\
( \pm 0,01)\end{array}$ & 100 & $\begin{array}{c}32,4 \\
( \pm 2,55)\end{array}$ & $\begin{array}{c}0,12 \\
( \pm 0,01)\end{array}$ & 30 & $\begin{array}{c}33 \\
( \pm 0,00)\end{array}$ & $\begin{array}{c}0,37 \\
( \pm 0,46)\end{array}$ \\
\hline 3 & GRA & 100 & $\begin{array}{c}16,2 \\
( \pm 6,49)\end{array}$ & $\begin{array}{c}0,04 \\
( \pm 0,03)\end{array}$ & 100 & $\begin{array}{c}19,2 \\
( \pm 6,39)\end{array}$ & $\begin{array}{c}0,06 \\
( \pm 0,04)\end{array}$ & 100 & $\begin{array}{c}22 \\
( \pm 6,45)\end{array}$ & $\begin{array}{c}0,08 \\
( \pm 0,03)\end{array}$ & 70 & $\begin{array}{c}22,3 \\
( \pm 19,72)\end{array}$ & $\begin{array}{c}0,09 \\
( \pm 0,03)\end{array}$ & 70 & $\begin{array}{c}28,4 \\
( \pm 15,80)\end{array}$ & $\begin{array}{c}0,10 \\
( \pm 0,02)\end{array}$ \\
\hline
\end{tabular}

Onde: $\mathrm{ABT}=$ abacate, $\mathrm{ACI}=$ açaí, $\mathrm{BCR}=$ bacuri, GRA = graviola. Os valores entre parênteses representam o desvio padrão da média. 

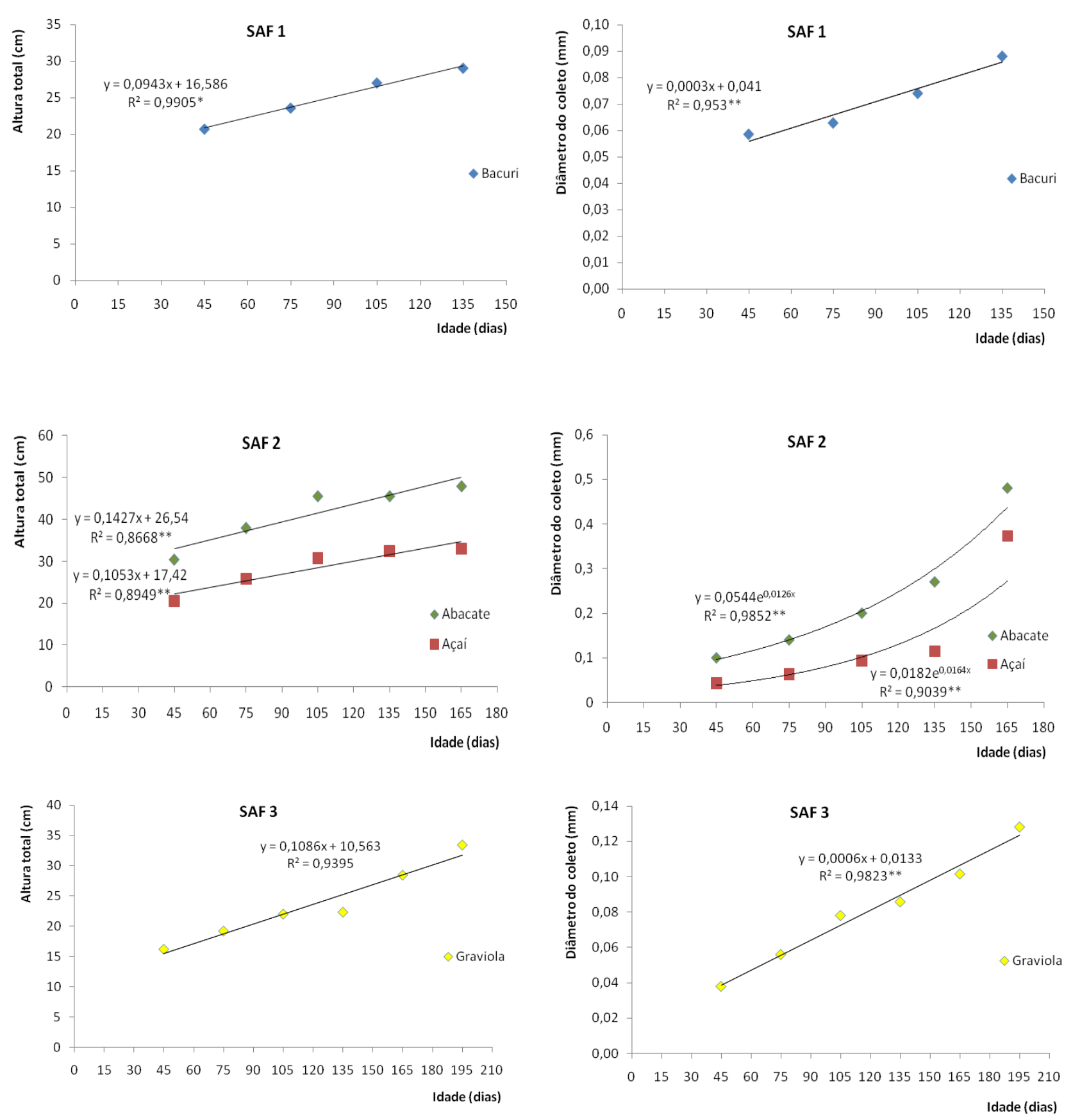

Figura 1. Relações dos parâmetros de crescimento altura total e diâmetro do coleto com a idade de plantio das espécies abacate, açaí, bacuri e graviola implantadas em ação-teste agrossivipastoril de unidade de produção familiar no P. A. Belo Horizonte I, São Domingos do Araguaia, Pará.

As práticas aplicadas pelos agricultores nas ações testes foram conduzidas de acordo com a realidade e disponibilidade de cada família para o desenvolvimento das ações-testes. Entre as restrições enfrentadas pelos agricultores para o estabelecimento das 
mudas frutíferas a campo pode-se relacionar: falta de adubação (química e/ou orgânica) e disponibilidade de mão de obra para realização das atividades exigidas (fabricação de composto orgânico, tratos culturais, limpeza e irrigação).

Apesar da baixa taxa de sobrevivência do açaí e do bacuri, e das condições adversas de implantação dos SAF considerou-se satisfatório o crescimento das frutíferas avaliadas em ações-testes agrossivilpastoris nas unidades de produção familiares do P. A. Belo Horizonte I até os 135 dias após o plantio.

Em ações futuras deve ser considerado o déficit hídrico da região durante o período seco do ano e alternativas que minimizem seus efeitos.

É importante ressaltar que este trabalho não teve como objetivo comparar as ações-teste agroflorestais e sim contribuir com informações sobre o desenvolvimento dessas espécies sob condições de implantação e manejo próprios dos sistemas produtivos diversificados e complexos de agricultores familiares do assentamento estudado

\section{CONCLUSÕES}

As espécies frutíferas açaí e bacuri tiveram a sobrevivência e crescimento comprometidos pelo estresse hídrico durante o período seco do ano. As espécies abacate e graviola se mostraram mais resistentes e, portanto mais indicadas a compor sistemas agroflorestais implantados em áreas de pastagens degradadas, nas condições avaliadas nas unidade de produção familiares no PA Belo Horizonte I.

\section{REFERÊNCIAS}

CALZAVARA, B. B. G. As possibilidades do açaizeiro no Estuário Amazônico. Belém: Boletim da Faculdade de Ciências Agrárias do Pará. 1972. 103 p.

CIAT. Centro Internacional de Agricultura Tropical. Cartillas para CIAL (Comités de Investigación Agrícola Local: $\mathrm{O}$ ensayo. Cali: CIAT/IPRA, 1993. 43 p.

CHIA, E.; DUGUÉ, P.; SAKHO-JIMBIRA, S. Les exploitations agricoles familiales sontelles des institutions? Agricultures, v. 15, n. 6, p. 498-505, 2006.

DUBOIS, J. C. L.; VIANA, V. M.; ANDERSON, A. B. Manual Agroflorestal para a Amazônia. Vol. 1. Rio de Janeiro, Brasil: REBRAF. 1996. 228 p.

FERNANDES, E. C. M.; SERRÃO, E. A. S. Protótipo e modelos agrossilvipastoris sustentáveis. In: SIMDAMAZÔNIA: SEMINÁRIO INTERNACIONAL SOBRE MEIO AMBIENTE, Pobreza e Desenvolvimento da Amazônia. Belém, 1992. Anais... Belém: Secretaria de Estado de Ciência, Tecnologia e Meio Ambiente, p.245251, 1992.

NAVEGANTES-ALVES, L. Gestion des pâturages en Amazonie Orientale: les décisions des pratiques. Montpellier, França: 
SUPAGRO - SIBAGHE, 2009 (Tese de doutorado em andamento).

OLIVEIRA, E. B.; SCHREINER, H. G. Caracterização e análise estatística de OLIVEIRA, T. K. Sistemas agroflorestais: vantagens e desvantagens. 1997. Disponível: <http://www.cpafac.embrapa.br/chefias/cna/a rtigos/sistagroflo.htm> Acesso em: 31/09/2009.

PEZO, D.; IBRAHIM, M. Sistemas Silvopastoriles. Turrialba, Costa Rica: CATIE, 1999. $276 \mathrm{p}$.

VEIGA, J. B.; PEREIRA, C. A.; MARQUES, L. C. T.; VEIGA, D. F. Sistemas Silvipastoris naAmazônia Oriental. Belém, Brasil: Embrapa Amazônia Oriental (Documentos, 56), 2000. 62 p.

XAVIER, G. P. L; SILVEIRA, A. J. Produção de sabão de sementes de frutos experimentos de agrossilvicultura. Boletim de Pesquisa Florestal. Curitiba, v.15, p.1940, 1987.

regionais. Revista Virtual de Iniciação Acadêmica da UFPA. Belém, v. 1, n. 1, p. 4. 2001.

\section{AGRADECIMENTOS}

O presente trabalho foi realizado com o apoio da Fundação de Amparo a Pesquisa do Estado do Pará - FAPESPA da PROPESP/UFPA através do PIBIC/UFPA e PIBIC/PARD. 